\title{
L'aménagement Écologique au Service de l'environnement : Cas de la Ville de Gabes et de M'Torrech (Sud Est Tunisien)
}

\author{
Docteur Saied Saoussen \\ Enseignant-Chercheur, Institut Supérieur Agronomique de Chott Mariem- \\ IRESA, Université de Sousse, Tunisie
}

\section{Docteur Lahmar Karim}

Enseignant-Chercheur, Université de Monastir, Tunisie

Laboratoire de recherche : Durabilité de l'oléiculture et l'arboriculture face aux changements climatiques, Institut de l'olivier Tunisie

Doi:10.19044/esj.2021.v17n14p300

Submitted: 24 February 2021

Accepted: 21 April 2021

Published: 30 April 2021
Copyright 2021 Author(s)

Under Creative Commons BY-NC-ND

4.0 OPEN ACCESS

Cite As:

Saoussen S. \& Karim L. (2021). L'aménagement Écologique au Service de l'environnement : Cas de la Ville de Gabes et de M'Torrech (Sud Est Tunisien). European Scientific Journal, ESJ, 17(14), 300. https://doi.org/10.19044/esj.2021.v17n14p300

\section{Resume}

La ville de Gabès (sud est Tunisien) est parmi les zones les plus touchées par une forte anthropisation. Les formes de pollutions atmosphériques ou continentales engendrées par les populations de cette ville, incitent à penser à des formes de corrections, à travers la proposition d'un éventuel projet d'aménagement écologique dans lequel l'environnement serait pris en compte. Cette étude propose une approche d'aménagement écologique en guise de modèle pour les aménagements. La méthodologie adoptée est basée premièrement sur une étude documentaire suivie d'une analyse des données. Puis, des logiciels de conception en $2 \mathrm{~d}$, de modélisation en $3 \mathrm{~d}$ et de cartographie ont été utilisés pour concevoir un plan d'aménagement écologique. Il ressort de cette étude que la pollution anthropique causée essentiellement par le Groupe Chimique Tunisien (GCT) de la localité de Gabès-ville et de M'Torrech engendre un danger potentiel sur la population (soit des maladies dermatologiques, respiratoires et allergéniques). Le plan d'aménagement écologique proposé aiderait les localités en question à diminuer l'effet de cette pollution. Les résultats mettent l'accent sur le danger de la pollution anthropique dans la région. 
Mots clés : Aménagement écologique, Environnement, Gabès

\title{
The Ecologic Landscaping at the Service of the Environment: Case of Gabes and M'Torrech (South-East of Tunisia)
}

\author{
Docteur Saied Saoussen \\ Enseignant-Chercheur, Institut Supérieur Agronomique de Chott Mariem- \\ IRESA, Université de Sousse, Tunisie \\ Docteur Lahmar Karim \\ Enseignant-Chercheur, Université de Monastir, Tunisie \\ Laboratoire de recherche : Durabilité de l'oléiculture et l'arboriculture face \\ aux changements climatiques, Institut de l'olivier Tunisie
}

\begin{abstract}
The city of Gabes (south-east of Tunisia) is among the areas most affected by strong anthropization. The forms of pollution recorded either atmospheric or continental encourage us to think better about forms of corrections. This could be in the form of an eco-landscaping project. The objective of this work is to provide a form of reflection on the possibility of intervening by setting up a project in which the environment is taken into consideration. In this study we try to focus on the present situation in relation to this issue. The adopted methodology is based firstly on a documentary study followed by data analysis. Then we try to come up with a design for an ecolandscaped park using photography, $2 \mathrm{~d}$ design, $3 \mathrm{~d}$ modeling and mapping software. Results obtained gave a more sustainable eco-landscaped development model. It emerges from this study that anthropogenic pollution caused mainly by the Tunisian Chemical Group (GCT) of the locality of Gabès-ville and M'Torrech generates a potential danger for the population (dermatological, respiratory and allergenic diseases). The proposed ecological development plan would help the localities in question to reduce the effect of this pollution. The results highlight the danger of anthropogenic pollution in the region.
\end{abstract}

Keywords: Eco-landscaping, Environment, Gabes

\section{Introduction}

Le besoin d'espace vert n'est pas à démontrer en milieu urbain, il ne relève pas seulement des enjeux esthétiques et paysagers, mais aussi des enjeux environnementaux ou écologiques. La présence de cet espace vital est indispensable au bien-être des populations surtout au niveau des villes qui souffrent de la dégradation de leur environnement. C'est le cas de la ville de 
Gabès située au sud est de la Tunisie qui depuis des années, souffre de la pollution causée par le secteur industriel. Les différentes recherches menées sur cette problématique (Particip Gmbh et ETI Consulting, 2017, Larbi K, 2017, Ben Nsir M, 2019; WMC, 2019 ; Blaise L, 2020, Khadimallah M, $2020 \ldots$... démontrent que la pollution engendrée essentiellement par le Complexe du Groupe Chimique Tunisien (GCT) a été de type atmosphérique, continentale et maritime. Les gaz des usines causent la pollution atmosphérique, les rejets des résidus des phosphates entrainent une forte mutation de la composition du sol et les navires de transport des produits de GCT induisent une pollution maritime. Cette pollution a des retombées nuisibles sur les secteurs économiques et a inscrit des pertes considérables aussi bien matérielles que sanitaires. En effet, plus de 95\% de la pollution atmosphérique provient des usines du Groupe Chimique Tunisien GCT (Medhioub, 2003). Le coût annuel de dégradation de l'environnement (CDE) est très élevé. Il représente, en 2015, plus que $27 \%$ de la valeur ajoutée (VA) de toutes les unités de production du GCT. Cette recherche vise à trouver des solutions au problème de la pollution à travers un choix judicieux des scènes à aménager, des matériaux écologiques et des plantes dépolluantes dans Gabès- ville et M'Torrech. L'approche paysagiste reste importante car elle permet de réfléchir à l'écologie du paysage urbain comme une véritable solution qui permet de répondre aux besoins des habitants en termes de qualité d'environnement et d'embellissement du cadre de vie, puis on pourrait améliorer la vie socioéconomique de la région: soit avec un nouvel investissement touristique ou par la création des nouvelles zones attractives (Larramendy et al ; 2014).

\section{Matériel et méthodes}

\subsection{Site d'étude : Gabès et ses alentours}

Le gouvernorat de Gabés est situé au sud-est de la Tunisie $\left(33^{\circ} 53^{\prime}\right.$ nord, $10^{\circ} 07^{\prime}$ est) et s'ouvre sur la Méditerranée par son golfe (figure 1).Il est situé à 410 Kilomètres de Tunis et couvre une superficie de $7175 \mathrm{~km}^{2}$, soit 4,4\% de la superficie du pays. Il est limité à l'Est par la Méditerranée (une côte de $80 \mathrm{~km}$ ), à l'Ouest par le gouvernorat de Kébili, au Nord-Est par le gouvernorat de Sfax, au Nord-Ouest par le gouvernorat de Gafsa et au Sud par le gouvernorat de Médenine. Le site d'etude comprend aussi la commune de M'Torrech qui appartient elle-même au gouvernorat de Gabès. 


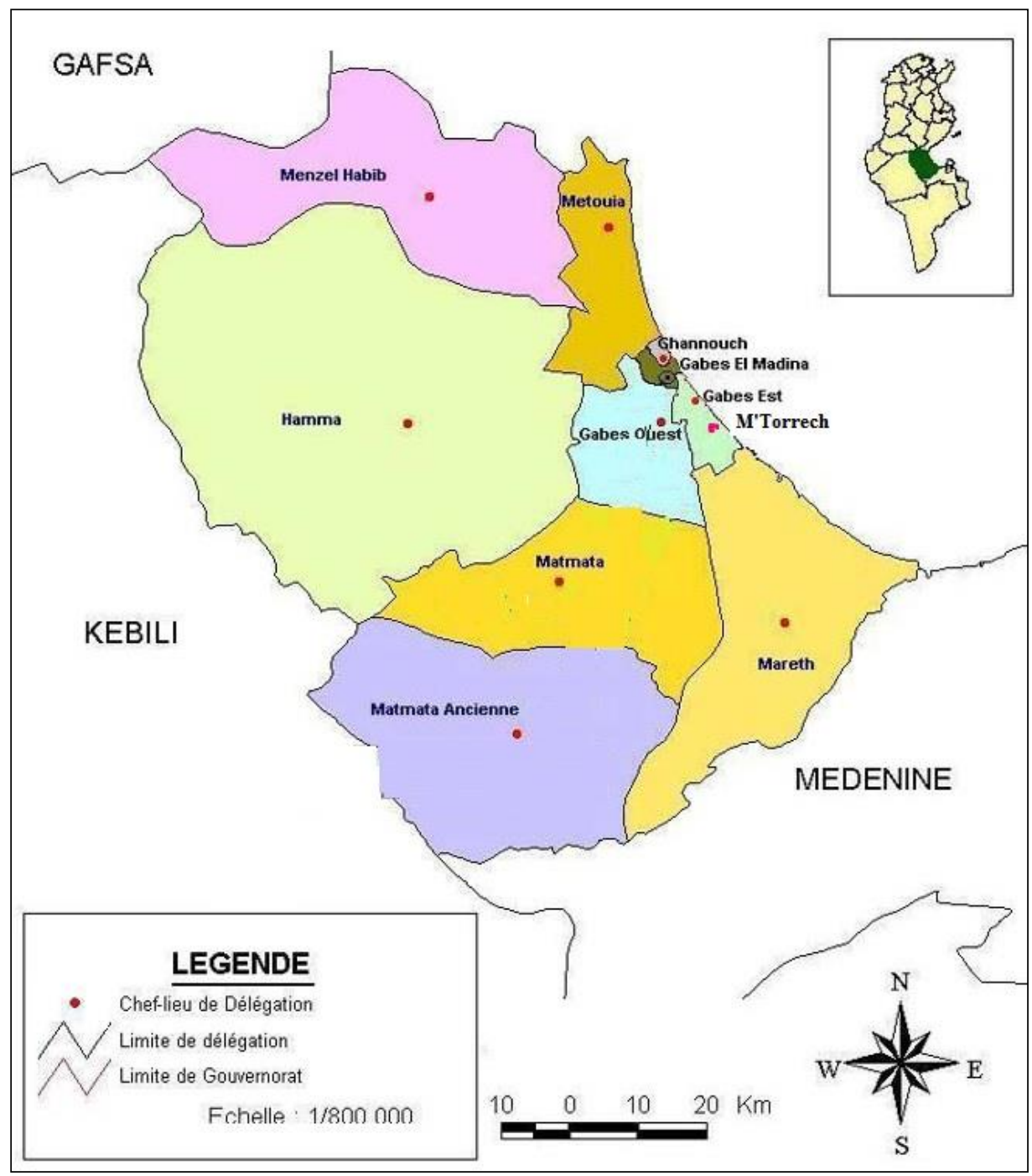

Figure 1: Localisation du gouvernorat de Gabès

\subsection{Méthodologie de travail}

Comme démarche méthodologique, cette recherche s'est basée, en premier lieu, sur une recherche documentaire sur la ville de Gabès permettant d'élargir le champ de connaissances de ses caractéristiques et spécificités. En deuxième lieu, deux diagnostics paysagers ont été effectués. Le premier a permis de comprendre les origines de la pollution et son impact sur l'environnement et l'économie de la ville de Gabès. Le second est que la commune de M'Torrech fait partie, comme on a déjà mentionné dans l'introduction, du gouvernorat de Gabes. On essaie de la prendre comme exemple pour proposer une approche d'aménagement écologique d'un parc urbain de la ville. Cet exemple peut être appliqué au niveau de tous les espaces 
verts de la ville. Le choix du site se justifie par sa vocation comme étant un parc urbain écologique riche en biodiversité et abritant une faune et flore à préserver et à valoriser. Pour la conception du plan d'aménagement proposé, la photographie aérienne du milieu d'étude, les logiciels de conception en $2 \mathrm{~d}$ (AutoCad), de modélisation en 3d (AutoCad 3d, SketchUp et Lumion) et de cartographie Arcgis (pour le géoréférencement des cartes) ont été utilisés. Ils ont permis de mener les analyses spatiales, les diagnostics paysagers de la ville de Gabès et du parc, de visualiser l'allure générale du projet (en 2d), ainsi que les croquis d'ambiances futures des scènes proposées (en 3d). Les visites de terrain ont quant à eux permis de mieux analyser le site et surtout de faire des prospections de terrain.

\section{Résultats}

\subsection{Gabès et M'Torrech : lieux propices pour une correction écologique}

Le gouvernorat de Gabès abrite un important pôle industriel spécialisé dans les industries chimiques, de traitement du phosphate, essentiellement, les industries agroalimentaires et les industries des matériaux de construction. Il comporte aussi d'importantes installations de production d'électricité et de gaz naturel (Le gisement Nawara), une cimenterie, des entreprises étrangères opérant essentiellement dans les industries manufacturières. A Gabès- ville et ses aloutours, (soit : M'Torrech, Matmata et Mareth), l'agriculture reste aussi un important pilier économique avec une superficie agricole estimée à 599267 ha (Particip Gmbh et ETI Consulting, 2017). La région renferme aussi une palmeraie de 300 mille palmiers dattiers et se spécialise également dans les cultures géoserricoles, produisant des primeurs destinées totalement à l'exportation (CRDA, 2020). Il est aussi connu pour ses richesses agricoles dans ses oasis telles que les cultures maraîchères, les grenades, les arbres fruitiers, les dattes et les cultures céréalières (CRDA, 2020).

De même, bien que le manque d'investissement et l'implantation de l'industrie chimique dans la région ont écarté toute opportunité de créer une industrie touristique sur le littoral, le tourisme est toujours en développement dans la ville vu la diversité de son paysage (la ville, la mer, les montagnes, 1'oasis et le désert). On note que Gabès- ville et M'Torrech restent des zones à fort potentiel touristique qui abritent un tourisme balnéaire. Ils possèdent des vastes plages sablonneuses sans rochers $(40 \mathrm{~km})$ et des oasis uniques au monde. Grace à ses spécificités naturelles et paysagères exceptionnelles, d'autres formes de tourisme en relation avec le tourisme alternatif ont eu lieu, tel que l'écotourisme et le tourisme de montagne, qui s'appuie sur l'architecture typique de la région et le mode de vie traditionnel préservés; le tourisme médical qui trouve aussi sa place grâce aux eaux thermales, à la fois chaudes et riches en minéraux, le tourisme saharien grâce à son ouverture au 
Sahara et le tourisme culturel avec ses nombreux monuments berbères, romains et islamiques ainsi que la diversité des produits traditionnels de l'artisanat. Malgré ce potentiel, le tourisme à Gabès et $\mathrm{M}$ 'Torrech reste un tourisme de passage (Municipalité de Gabès, 2020).

\subsection{Proposition d'une nouvelle approche d'aménagement écologique}

Selon Larramendy S et al (2004), La conception des espaces publics écologique doit tenir compte des différents enjeux écologiques existants et qualité du paysage urbain. (Larramendy $S$ et $a l ; 2014$ ). En se basant sur cette réflexion, on peut dire que la ville de Gabès et ses alentours possèdent des facteurs qui pourront être l'issue d'une correction écologique et environnementale. Les figures 4 et 5 , ci-dessous, montrent la situation géographique de la commune de $\mathrm{M}$ 'Torrech par rapport à Gabès ainsi que la situation du parc par rapport à la ville de M'Torrech.

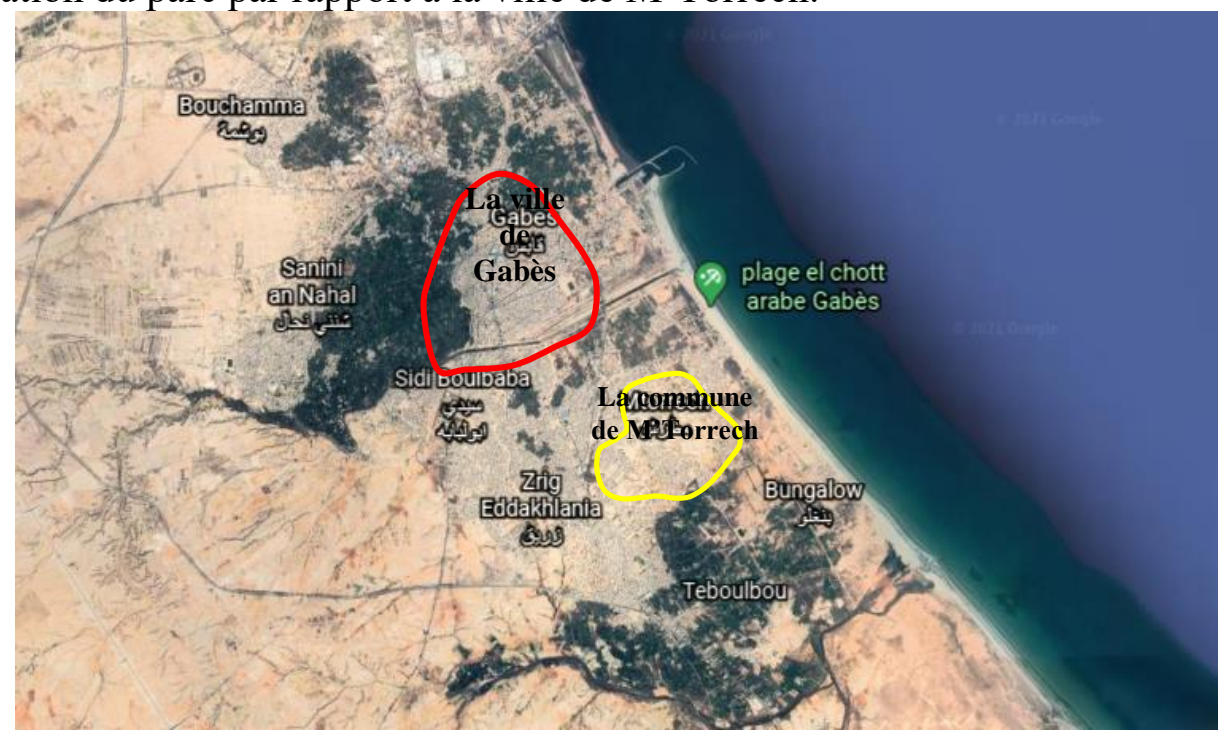

Figure 2 : Photographie aérienne montrant l'emplacement géographique de la commune de M'Torrech par rapport à la ville de Gabès

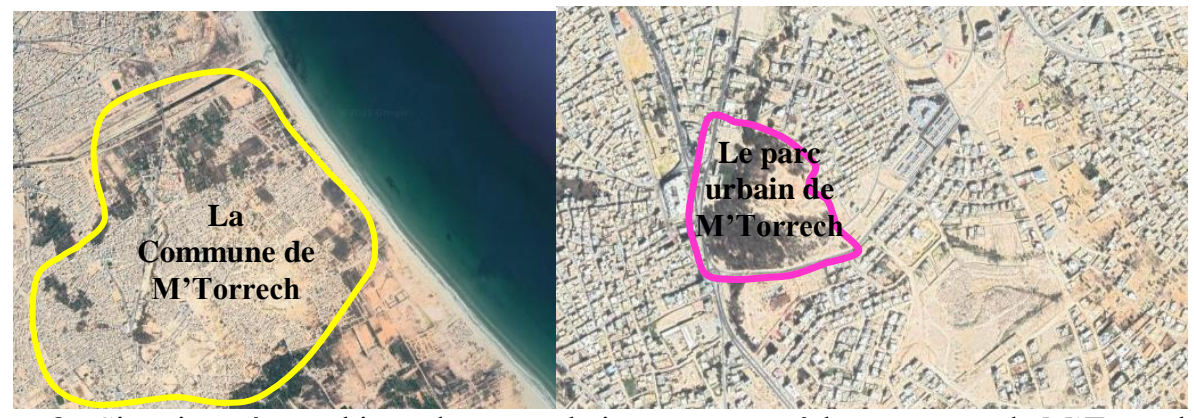

Figure 3 : Situation géographique du parc urbain par rapport à la commune de M'Torrech 
Le site qui occupe une superficie de $13 \mathrm{Ha}$, se trouve à environ $3 \mathrm{~km}$ au Sud-ouest du Centre-ville de Gabès et occupe le sommet d'une colline, d'une altitude comprise entre 32 et $45 \mathrm{~m}$ environ. Il se caractérise par une topographie exceptionnelle offrant des vues panoramiques de forte importance. Il est entouré par des quartiers polyfonctionnels ou on enregistre une forte concentration d'équipements publics.

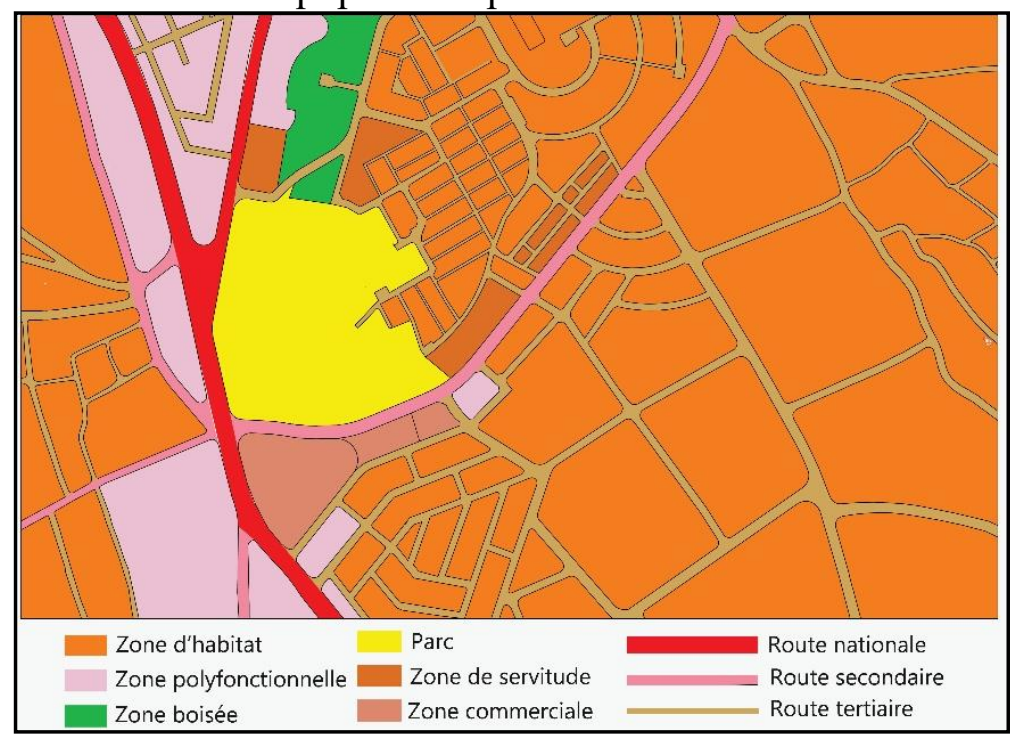

Figure 4: Croquis d'équipement de la commune de M'Torrech

Il faut penser à un aménagement de continuité et de relais entre les différentes entités entourant le site tout en assurant une bonne intégration paysagère dans la région (figure 6). Ce site est aussi à vocation sociale puisqu'il est demandé par les habitants de la cité. Le site d'étude est aussi en relation avec ses alentours. Donc pour mieux comprendre les différents types d'usager, il faut élaborer une identification de l'ensemble de ses composantes limitrophes et environnantes. D'après la carte ci-dessous, toutes les entités paysagères aux alentours du site sont repérées. 


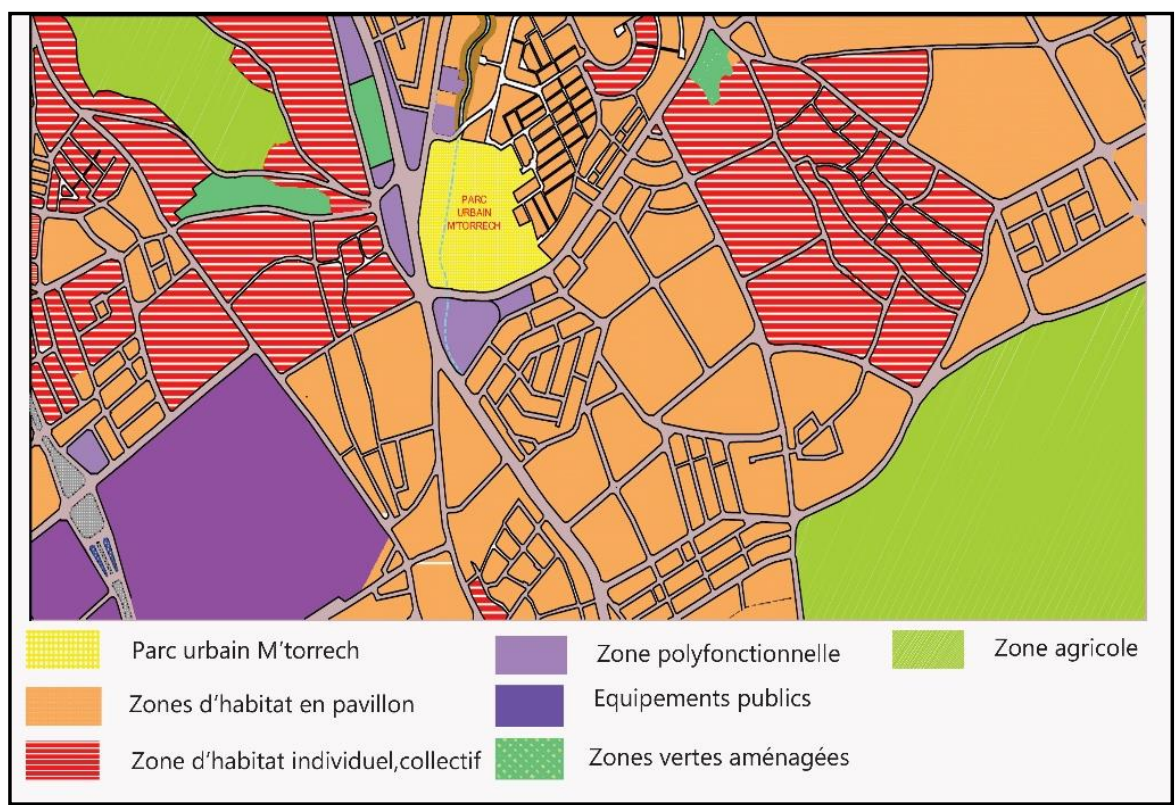

Figure 5: Carte des entités paysagères du site (PAU 2009)

On remarque que le site se trouve dans une zone purement urbaine et on constate l'absence totale des espaces verts aménagés pour les habitants, donc il faut penser à un aménagement qui injecte de la verdure au sein de la ville (figure 8). La figure ci-dessous montre aussi que le site est dominé par les formes régulières des bâtiments. Pour adoucir ces formes rigides, l'aménagement écologique doit être fluide, souple et inspiré de l'écosystème oasien de la région de Gabès. 


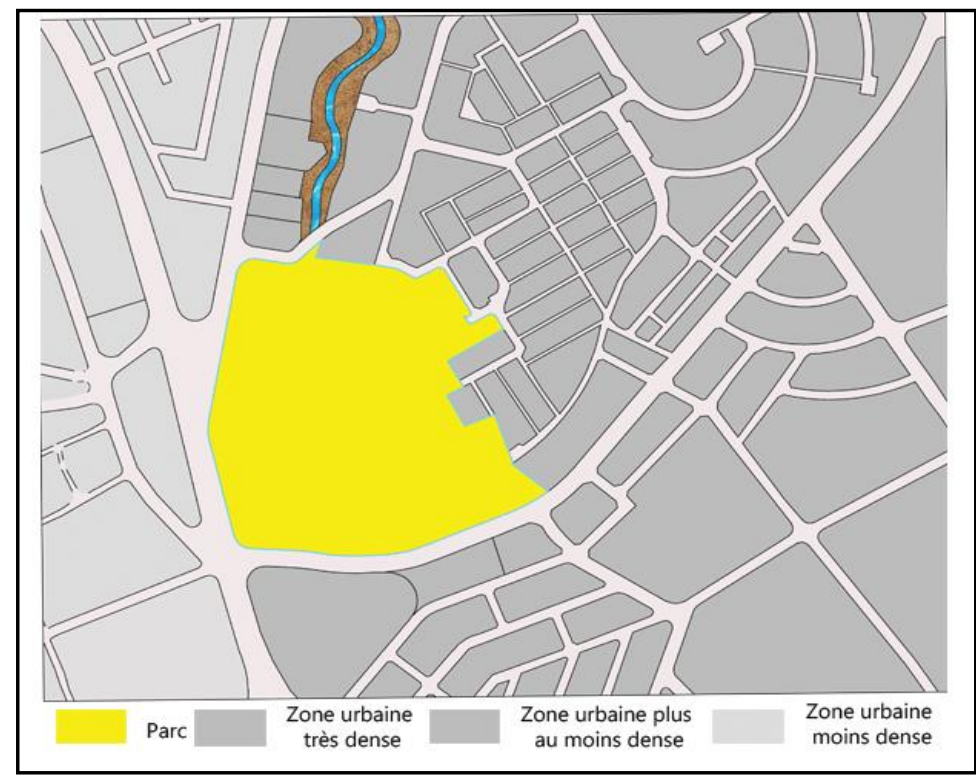

Figure 6: Les formes existantes aux alentours du site (PAU 2009)

Sur le plan naturel, le parc se caractérise par des spécificités naturelles particulières. Le relief occupe le haut d'une colline et la vallée du cours d'eau de Gabès. Il se singularise par un ravinement important occupant près de 50 $\%$ de la surface du parc. Il culmine en deux parties, l'une autour d'un château d'eau situé à la limite Nord-est du parc, l'autre à la limite Sud-Est ou les hauteurs sont accentuées par les constructions limitrophes. Au pied de ces deux collines, des banquettes ou «tabias » sont aménagées parallèlement aux courbes de niveaux pour préserver le sol de l'érosion, elles sont souvent accompagnées par des pistes de desserte ou des traversées du parc. Il est aussi occupé par un grand ravin large et à fond plat qui descend vers le cours d'eau de Gabès et constitue le drain principal des eaux pluviales des quartiers voisins. La largeur de ce ravin varie de cinq à quelque dizaine de mètres. Des seuils transversaux en gabions sont aménagés tous les cinquante mètres environ afin de retenir l'eau et contenir le torrent, et qui, faute d'entretien, commençant par endroit à subir quelques dommages. Comme ressources hydrauliques, on note la présence d'un château d'eau dans les limité NordEst. Le site se trouve aussi riche en palette végétale, c'est une forêt d'Eucalyptus très touffue et de garrigue. Les végétaux existants souffrent à la fois du manque d'eau et d'entretien. En effet, pour un aménagement paysager durable et fonctionnel, l'Eucalyptus sera un mauvais choix de plantation puisqu'il est considéré comme une pompe d'eau et surtout dans la ville du Gabès qui souffre énormément du manque d'eau potable et de précipitation. D'où dans le plan d'aménagement il faut choisir une palette végétale qui 
résiste à la sécheresse et qui contribue à la purification de l'air comme les palmiers (figures 9 et 10).

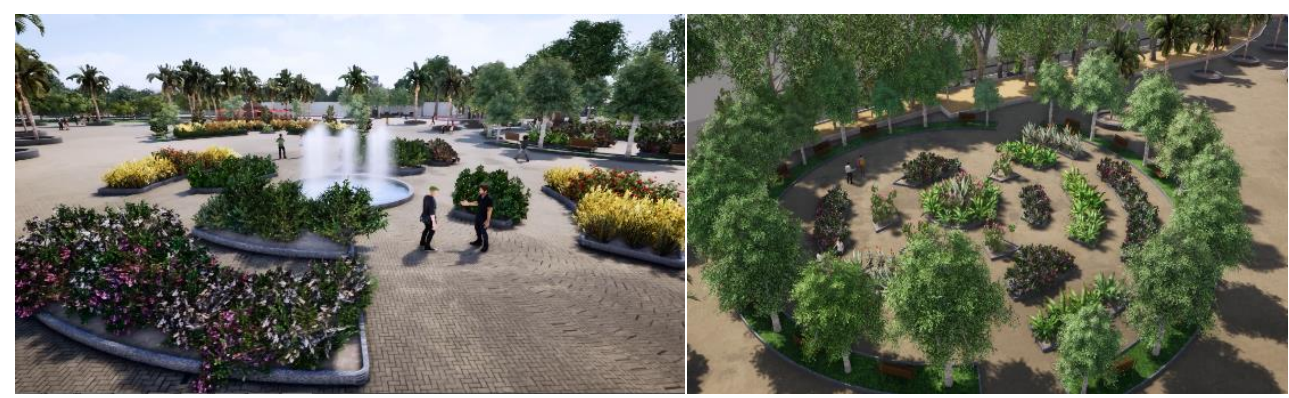

Figure 9 : Jardin aromatique et jardin exotique

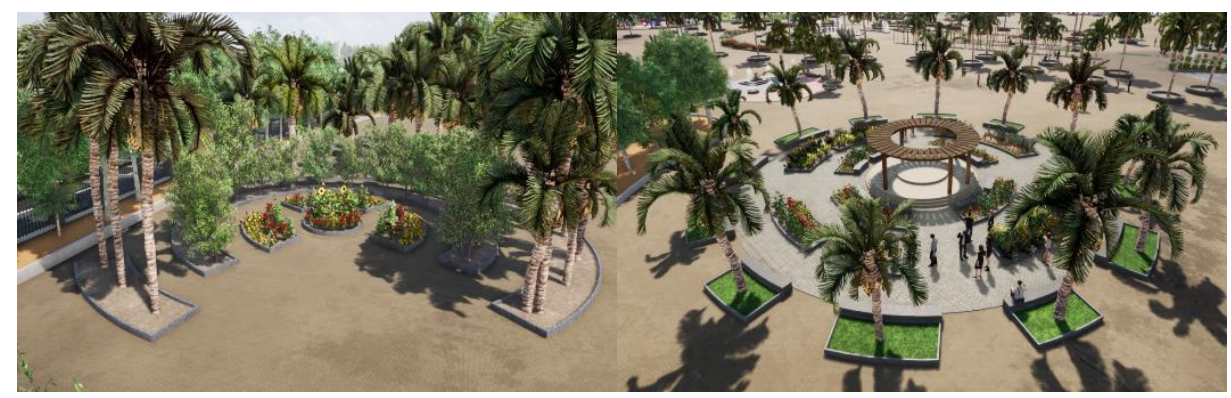

Figure 10 : Jardin Oasis et jardin des pratiques agricoles

L'aménagement est une proposition d'un modèle de conception qui servira dans le conditionnel d'atténuer les impacts de la pollution. La mise en place d'un service d'entretien et de suivi ne peut que améliorer les attente de ce type de projet dans la lutte contre les effets nuisibles dela pollution. Par ailleurs, après l'exécution des travaux, un suivi et une gestion seriont effectués pour garantir sa durabilité. De même des instruments de mesure de pollution nous permettront de comparer les résultats obtenus aux anciens résultats et par la suite évaluer le degré d'efficacité du projet proposé dans l'amélioration de l'environnement.

\section{Discussion}

Une décision de délocalisation des unités polluantes de Gabès a été prise par les autorités publiques le 29 Juin 2017 mais en vain, rien n'est fait jusqu'à maintenant (Blaise L, 2020). Des projets écologiques ont été aussi proposés tel que le projet de "L'oasis intelligente ». Ce projet conçoit un quartier écologique au centre urbain qui relie la mer, l'oasis et le canal de Gabès. C'est une tentative de revaloriser l'oasis et l'introduire au sein de la ville pour diminuer les effets de l'urbanisation et lui redonner aussi la vocation de l'oasis marine. Une étude stratégique a été menée afin d'évaluer la 
faisabilité du projet (Khadimallah M, 2020). Un autre projet a eu aussi lieu, il s'intitule « Madinatouna » et s'inscrit dans le cadre du « Programme Pays de Cites Alliance ». Il vise à promouvoir les initiatives locales de planification urbaines stratégiques (Municipalité de Gabès, 2020). Cette prise de conscience de la nécessité d'injecter de la verdure au sein des centres urbains représente un atout indéniable en termes de preservation de l'environnement surtout que la décision de la délocalisation des sites polluants doit s'engager dans une politique de responsabilité sociale et environnementale qui permette de prévenir réellement la pollution. Les espaces végétalisés urbains constituent aujourd'hui pour la ville des marqueurs qualitatifs et un moyen d'assurer l'éducation à l'environnement et au développement durable (Bourcier A., 2012). Pour cela, la proposition de l'aménagement écologique du parc urbain de la ville de M'Torrech a pour but de diminuer l'effet de la pollution à travers un choix judicieux d'espèces dépolluantes et tolérant les conditions du milieu. De même, l'utilisation des matériaux écologiques durables, l'exploitation des eaux de pluie et le recours aux énergies renouvelables surtout que la ville est doté d'un potentiel solaire important (2300 à $\left.2400 \mathrm{kWh} / \mathrm{m}^{2} / \mathrm{an}\right)$ sont aussi prioritaires (Particip Gmbh et ETI Consulting, 2017). Comme programme d'aménagement, le parc comportera différentes composantes et scènes paysagères qui visent à rendre l'espace fonctionnel, accueillant et qui contribuent à préserver ses spécificités naturelles et sa vocation écologique. Il comportera des espaces d'accueil et de détente, des aires de jeux pour enfants, une pépinière et des parcelles de cultures pour cultiver les plantes dépolluantes, et différents types de jardins qui renforcent le côté écologique du milieu jardins tels que le jardin aromatique, le jardin exotique, le jardin des pratiques agricoles et une serre vitrée.

\section{Conclusion}

Cette recherche propose une nouvelle approche de réaménagement écologique qui prend en compte la dimension transversale des thèmes urbains, économiques, sociaux, culturels, environnementaux et de gouvernance, tout en veillant à prendre en considération les spécificités et la fragilité des écosystèmes de la ville. Cette approche éco paysagère se pose comme étant une solution qui permettra de redonner une autre vie au parc, actuellement délaissé, et de créer un espace écologique, agréable à vivre et aussi multifonctionnel... Afin de garantir les meilleures conditions pour le déroulement du projet, le gouvernement doit fournir des garanties suffisantes concernant la mobilisation des fonds nécessaires pour la réalisation du projet.

\section{References :}

1. Ben Nsir, M., (2019). Pollution dans la région du Gabès ; le prix fort d'une industrie mal pensée. Publié le 27 Février 2019 in 
https://www.leconomistemaghrebin.com/2019/02/27/pollution-dansla-region-de-gabes-le-prix-fort-dune-industrie-mal-pensee/

2. Bourcier, A., (2012). Le paysage au service de la biodiversité dans la ville durable, VertigO - la revue électronique en sciences de l'environnement [En ligne], Hors-série 14 | septembre 2012, mis en ligne le 15 septembre 2012.

3. Blaise, L., (2020). Les habitants meurent à petit feu: en Tunisie, le phosphate pollue l'air de Gabès. Publié le 2 janvier 2020.

4. Gramond, D., (2020). La ville durable, un territoire d'avenir pour la biodiversité urbaine ?.Projets de paysage [En ligne], 13 |2015, mis en ligne le 31 décembre 2015, consulté le 16 février 2021. URL: http://journals.openedition.org/paysage/9791

5. Khdimallah, M., (2020). Tunisan smart citeies, Gabes, l'Oasis intelligente. Publié le 18 mars 2020 in https://lapresse.tn/53472/gabesloasis-intelligente/

6. Larbi, K., (2017). En Tunisie, Gabès lutte contre la pollution industrielle. Publié le 21 Aout 2017

https://www.lantenne.com/En-Tunisie-Gabes-lutte-contre-lapollution-industrielle_a38517.html

7. Larramendy, S., Huet, S., Micand, A.,\&Provendier, D., (2014). Conception écologique d'un espace public paysager. Guide méthodologique de conduite de projet, plante \& cité, Angers, 94 p.

8. Medhioub, K., (2002).Impact des rejets atmosphériques du complexe chimique de Gabès sur les parcelles agricoles de Chott Essalem et Bou Chemma et Ghannouch. Expertise demandée par le tribunal de Gabès :84pp.

9. Municipalité de Gabès, (2020), Stratégies de développement durable de la ville de Gabès 2030, in rapport de synthèse de la SDV de Gabès.

10. Particip Gmbh \&ETI Consulting., (2017). Etude d'impact de la pollution industrielle sur l'économie de la région de Gabès. In Rapport final présenté le 30 Décembre 2017.

11. WMC, (2019). Environnement : Les industries polluantes à Gabès en débat. Publié le 1 mars 2019. https://www.webmanagercenter.com 